Development of HPLC-UV method for rapid and sensitive analysis of topically applied tetracaine: its comparison with a CZE method

\begin{abstract}
ARTICLE in Biomedical Chromatography. 06/2014; 28(6):826-30.
\end{abstract}

DOI: 10.1002/bmc.3150 


\section{Development of HPLC-UV method for rapid and sensitive analysis of topically applied tetracaine: its comparison with a CZE method}

Faisal Al-Otaibi ${ }^{1}{ }^{\dagger}$, Essam Ghazaly ${ }^{2} \uparrow *$, Atholl Johnston ${ }^{1}$ and David Perrett $^{3}$

${ }^{1}$ Clinical Pharmacology, William Harvey Research Institute, Queen Mary University of London, Charterhouse Square, London, EC1M6BQ, UK

${ }^{2}$ Centre for Haemato-Oncology, Barts Cancer Institute, Queen Mary University of London, Charterhouse Square, London, EC1M6BQ, UK

${ }^{3}$ Bioanalysis, William Harvey Research Institute, Queen Mary University of London, Charterhouse Square, London, EC1M6BQ, UK

*Correspondence to: Essam Ghazaly, Centre for Haemato-Oncology, Barts Cancer Institute, Queen Mary University of London, Charterhouse Square, London, EC1M6BQ, UK. E-mail: e.a.ghazaly@qmul.ac.uk

${ }^{\dagger}$ These authors contributed to the work equally and should be regarded as co-first authors 


\begin{abstract}
Topically applied tetracaine is a local anaesthetic. A novel HPLC method for rapid and sensitive analysis of tetracaine was developed and compared with a short end direction capillary zone electrophoresis (CZE) method (Al-Otaibi, Tucker, Johnston and Perrett 2009). The method was developed and validated for the separation and quantification of tetracaine in skin samples removed by "tape stripping". Tetracaine was extracted from tape with 100\% methanol, which was then diluted to $50 \%$ with water for injection. Tetracaine and internal standard, procaine, were separated, on a reversed phase Luna $\operatorname{PFP}(2), 3 \mu \mathrm{m}, 150 \mathrm{~mm} \times 4.6$ mm column at ambient temperature using isocratic elution with $\mathrm{KH}_{2} \mathrm{PO}_{4}$ buffer $(\mathrm{pH} 2.5)$ and methanol $(35: 65, \mathrm{v}: \mathrm{v})$. The flow rate was $1 \mathrm{ml} / \mathrm{min}$, with detection at $312 \mathrm{~nm}$. The limit of quantification for tetracaine was $0.03 \mu \mathrm{g} / \mathrm{ml}$. Calibration lines were linear with $\mathrm{r}^{2}$ values greater than 0.99. Within and between assay imprecision and the percentage of inaccuracy for the QC samples including lower and upper limits of quantitation were $<6 \%$ and $<10 \%$. The absolute mean recovery of tetracaine was $>92 \%$. Compared to CZE, the mean percentage error and the absolute mean percentage error was 0.62 and 6.29. Both methods were compared in a number of pharmacokinetic studies.
\end{abstract}




\section{Introduction}

Tetracaine (amethocaine, 2-dimethylaminoethyl 4-butylaminobenzoate, $\mathrm{C}_{15} \mathrm{H}_{24} \mathrm{~N}_{2} \mathrm{O}_{2}$. $\mathrm{HCl}$ ), is a potent local anaesthetic of the amino ester group, and is used for topical anaesthesia in ophthalmology, spinal anaesthesia and nerve block. It is also the first alternative anaesthetic for nasal septoplasty since cocaine (Drivas, Hajiioannou, Lachanas, Bizaki, Kyrmizakis and Bizakis 2007). Tetracaine, as the hydrochloride, can be formulated in solutions, creams, gels, and as the base in ointments. The topical formulation of tetracaine gel as a local anaesthetic was developed in the early 1990s, enabling a more rapid and long duration of action than the mixture of lidocaine and prilocaine, known as EMLA cream (Bishai, Taddio, Bar-Oz, Freedman and Koren 1999, Boyd and Jacobs 2001, Martindale, Reynolds and Parfitt 1996, McCafferty, Woolfson and Boston 1989, Woolfson, McCafferty and Boston 1990). The onset of action is 30 to 45 minutes, while EMLA cream is about 1 hour, without any risk of methemoglobinaemia.

HPLC with UV detection has been used for determination of tetracaine in plasma (Mazumdar, Tomlinson and Faulder 1991, Menon and Norris 1981, Murtaza, Jackman, Alexander, Lleshi-Tali, Winnie and Igic 2001, Qin, Jiao, Zhong, Shi, Zhang, Li and Cui 2010) and was capable of detecting tetracaine and its major metabolite (Figure 1). All of these methods have relied upon ion-pairing agents in conjunction with ODS C18 stationary phase to achieve good separation for tetracaine although peak shape could still be problematical. In an earlier we reported a simple CZE method for tetracaine (Al-Otaibi, Tucker, Johnston and Perrett 2009).

In this study, a novel rapid and sensitive HPLC-UV method for analysis of topically applied tetracaine was developed using a pentafluoryl phenyl PFP(2) HPLC column that allowed fast analysis, sharp peak resolution without the need for ion-pairing agents. Thus, using simple and rapid techniques such as CE or HPLC-UV is possible as expensive techniques such as LC-MS are not required. In addition, HPLC techniques can be compared with CE to find out more appropriate and reliable methods. In this study, we validated the HPLC-UV method and compared it to previously published CZE-UV 


\section{Materials and methods}

\section{General chemicals}

Tetracaine hydrochloride ( $\mathrm{HCl}$; lot no. $065 \mathrm{k} 1501$; $99.9 \%$ purity) for calibrator and control sample preparation, 4-Butyaminobenzoic acid (Lot no 02514BJ; 97\% purity), tetracaine major metabolite, and procaine hydrochloride ( $\mathrm{HCl}$; lot no. $114 \mathrm{k} 0569 ; 100 \%$ purity), the internal standard were obtained from Sigma Aldrich (Poole, UK). HPLC-grade solvents were obtained from Rathburn Chemicals Ltd (Walkerburn, UK). All other AR grade reagents were obtained from Merck (BDH) Ltd (Poole, UK).

\section{Adhesive tape}

Samples were obtained by a tape stripping technique from the skin of healthy volunteers, who had received topical tetracaine. The adhesive tape used in this study was $19 \mathrm{~mm}$ width, Tesa 4205 PV5, Beiersdorf, Hamburg Germany, supplied by TESA UK. The concentration of the drug in such samples can be higher than that in plasma samples of topically applied drugs (Bareggi, Pirola and De Benedittis 1998).

\section{Chromatographic conditions and instrumentation}

A Shimadzu LC-6A pump, Gilson 231 autosampler fitted with a $200 \mu \mathrm{L}$ injector loop, Gilson diluter 401 coupled to a Shimadzu SPD-6A UV/Vis detector was used. Separations were carried out using a Luna PFP (2) column, $3 \mu \mathrm{m}, 150 \mathrm{~mm}$ x $4.60 \mathrm{~mm}$ from Phenomenex Macclesfield U.K. The injection volume was $10 \mu \mathrm{L}$. The analytes were eluted using isocratic elution at $1 \mathrm{~mL} / \mathrm{min}$ with $\mathrm{KH}_{2} \mathrm{PO}_{4}$ buffer $(\mathrm{pH} 2.5)$ and methanol (35:65, v:v) and were monitored by ultraviolet absorption at $312 \mathrm{~nm}$. The data acquisition system consisted of a data interface (HP 35900 interface) and HP Chemstation, version A.04.01. The software was operated under Windows 95 environment.

\section{Sensitivity}

The lower limit of detection was determined as the concentration of tetracaine that had a peak height three times higher than the baseline noise. The lower limit of quantification was defined as the lowest tetracaine concentration that can be quantified with a $\mathrm{CV}<10 \%$. 


\section{Recovery}

Recovery of tetracaine was calculated by dividing the peak area of the standard samples spiked with tetracaine by the peak area of the same tetracaine concentration in $50 \% \mathrm{MeOH}$.

\section{Stability}

The stability of tetracaine was determined using tape samples spiked with tetracaine at 100 $\mu \mathrm{g} / \mathrm{ml}$ stock solutions. Aliquots from this solution were injected into the HPLC after each freeze / thaw cycles stored at $-20^{\circ} \mathrm{C}$.

\section{Calibrators and quality control samples}

\section{Calibrator}

$50 \mathrm{ml}$ of tetracaine stock standard solution was diluted to $100 \mathrm{ml}$ with $50 \% \mathrm{v} / \mathrm{v}$ aqueous $\mathrm{MeOH}$ to produce a stock solution $(2.5 \mathrm{mg} / \mathrm{ml})$. Working calibration solutions were prepared by diluting the stock solution with $50 \% \mathrm{v} / \mathrm{v}$ aqueous $\mathrm{MeOH}$, as follow 0.03, 0.1, 0.3, 1, 3, 10, 30, $100 \mu \mathrm{g} / \mathrm{ml} .0 .03$ and $100 \mu \mathrm{g} / \mathrm{ml}$ were used as lower limit of quantitation (LLOQ) and upper limit of quantitation (ULOQ), respectively.

\section{Quality control $(Q C)$ samples}

A sub-stock (QC 4, $1.25 \mathrm{mg} / \mathrm{ml}$ ) was prepared. Working controls were prepared by diluting the sub stock with $50 \% \mathrm{v} / \mathrm{v}$ aqueous $\mathrm{MeOH}$, as follow $0.5,5,50 \mu \mathrm{g} / \mathrm{ml}$.

\section{Calculating inaccuracy and imprecision}

Inaccuracy was tested by the determination of low, medium and high quality control samples, together with the LLOQ and ULOQ samples. Each sample contained tetracaine. The nominal values for the low, medium and high control samples were $0.5,5,50 \mu \mathrm{g} / \mathrm{ml}$, respectively. The nominal values for the ULOQ and LLOQ were the same nominal concentration as the highest and the lowest calibration standards, respectively.

Assay imprecision was measured both within and between batch by the analysis of three control samples, the LLOQ and the ULOQ. Within-batch and between-batch imprecision, regression coefficient and the slope of the calibration line were calculated from the peak area 
ratio by excel 2007, using internationally agreed methods (ISO-5725 1994). The statistical analysis and graphical presentation used Excel 2007.

Extraction of tetracaine from tape samples was has previously described (Al-Otaibi, Tucker, Johnston and Perrett 2009).

\section{Results}

\section{HPLC Separation}

We tested four different HPLC columns (C8, C18, phenyl and PFP). The PFP column achieved the best separation and peak resolution for tetracaine and the internal standard procaine. Mobile phase $\mathrm{pHs}$ ranging from 2-8 were also studied and the optimum $\mathrm{pH}$ for retention was found to be 2.5 . (Figure 2)

\section{Sensitivity}

The limit of detection (LLOD) for tetracaine was $3 \mathrm{ng} / \mathrm{ml}$. The limit of quantification (LLOQ) was $30 \mathrm{ng} / \mathrm{ml}$.

\section{Specificity}

Error! Reference source not found.A shows a chromatograph of a time zero patient sample with no significant interfering peaks at the retention time of procaine $(150 \mu \mathrm{g} / \mathrm{ml})$ or tetracaine, compared with figure $2 \mathrm{~B}, 10 \mu \mathrm{g} / \mathrm{ml}$ tetracaine, and figure $2 \mathrm{C}, 1$ hour posttreatment chromatograph, at $\lambda_{\max }$ of $312 \mathrm{~nm}$.

\section{Linearity}

Figure 3 shows the calibration line containing the eight non-zero calibrators assayed. Nominal values were $0.03,0.10 .3,1,3,10,30,100 \mu \mathrm{g} / \mathrm{ml}$ tetracaine and $150 \mu \mathrm{g} / \mathrm{ml}$ procaine. Six batches of calibration curve were plotted using the area ratio of tetracaine to IS Vs known concentration of tetracaine. All the results were calculated using a $1 / \mathrm{x}^{2}$ weighted quadratic regression.

\section{Accuracy and precision}

Accuracy was tested by determination of low, medium and high quality control samples, together with the LLOQ and ULOQ samples. Each control sample contained tetracaine. The 
nominal values for the low, medium and high control samples were $0.5,5,50 \mu \mathrm{g} / \mathrm{ml}$ respectively. The nominal values for the ULOQ and LLOQ were the same nominal concentration as the highest and the lowest calibration standards, respectively.

Assay precision was measured both within and between batch by the analysis of three control samples, the LLOQ and the ULOQ.

\section{Within assay reproducibility}

For within and between batch precision the LLOQ and ULOQ, the three control samples were each assayed six times during one assay. The CV for imprecision and the percentage inaccuracy for all the quality control samples including LLOQ and ULOQ was below 10 and $5 \%$, respectively.

\section{Between assay repeatability}

The mean concentration from each assay was used to calculate the between assay reproducibility. The $\mathrm{CV}$ for imprecision and percentage inaccuracy for all the quality control samples including LLOQ and ULOQ was below 7 and 5\%, respectively.

Within and between batch precision was calculated using FDA agreed methods (FDA 2001).

\section{Stability}

The CV\% of $100 \mu \mathrm{g} / \mathrm{ml}$ tetracaine was less than $2 \%$, when injected into the HPLC after each freeze / thaw cycle.

\section{Recovery}

The absolute recovery of tetracaine ranged from 79 to $104 \%$. The result was obtained by dividing the peak height for specific tetracaine concentrations in methanol: water, 50:50 solutions and the peak height of the same concentration in skin tape stripping soaked in methanol:water, 50:50 from time zero (sample 0). 


\section{Method comparison}

Tape samples obtained from patients' skin were successfully analyzed by both the new HPLC method and the CZE technique (Al-Otaibi, Tucker, Johnston and Perrett 2009). The limit of quantification for the HPLC method for tetracaine was $0.03 \mu \mathrm{g}$ which was better than reported for the CZE method. The calibration lines for CE and HPLC were linear for $25-$ $1200 \mu \mathrm{g} / \mathrm{ml}$ and $0.03-100 \mu \mathrm{g} / \mathrm{ml}$, respectively and both had $\mathrm{r}^{2}$ values greater than 0.99 . The $\mathrm{CV} \%$ for both within and between assay imprecision and the percentage of inaccuracy for the quality control samples including lower and upper limits of quantitation for HPLC were $<6 \%$ and $<10 \%$, respectively, and for CE were $<12.1 \%$ and $<11 \%$, respectively. The shorter migration times obtained for tetracaine $(1.36 \mathrm{~min})$ are an advantage of $\mathrm{CE}$ compared to 3.75 min retention in HPLC. An internal standard was used to minimize injection volume variability, and error resulting from methanol evaporation in sample. The absolute mean recovery of tetracaine in HPLC was $>92 \%$, and in CE was $>97 \%$.

The two methods of measurement were compared to find out if there was any difference related to the analysis. The analyzed data were calculated and plotted for both methods, Figure 4 shows Bland-Altman analysis was in a range of $\pm 1.96 \mathrm{SD}$ from the mean, and Table 4 shows the mean, standard deviation, and standard error of the mean percentage error (MPE) and the absolute mean percentage error (AMPE) between the HPLC and the CE measurements.

\section{Discussion}

In this study an HPLC method was developed, optimized and validated for analysis of topically applied tetracaine. This is the first HPLC method to be developed for this purpose. The new HPLC method resulted in a good separation with excellent resolution and minimum peak tailing. There was no need for ion-pairing or gradient elution. The chromatography was fast, clean and ideally suited for PK studies when lots of samples are to be assayed. However it is difficult to rationalise the precise separation mechanism on a PFP column. PFP is known to exhibit both normal and reversed phase characteristics (Marin and Barbas 2006). The HPLC on a PFP column used in this study gave an unexpected separation with a short retention time of 2.6 and $3.8 \mathrm{~min}$ for procaine and tetracaine, respectively. All the validation results met the international requirements as outlined by the FDA's 2001 bioanalytical method validation guidelines (FDA 2001). 
HPLC and CE methods with UV detection have been compared for the determination of tape samples obtained from healthy volunteers given tetracaine as part of a pharmacokinetic study. UV detection was chosen to be used for its simplicity and low cost. Although the HPLC method was better in terms of sensitivity, both methods in this study measured all samples obtained without any problems, including the lower concentrations obtained in the tape samples. The data obtained were within $\pm 1.96 \mathrm{SD}$, and $17 \%$ error of the difference. Nevertheless the purpose of this study was to develop a fast and simple analytical method, to determine tetracaine in skin using tape samples from 12 healthy volunteers given tetracaine as part of a pharmacokinetic drug delivery study. Although the imprecision was higher, the lower operation costs and volume of electrolyte used, and faster running time made CE preferable to HPLC for this kind of study. This observation is in agreement to that of other authors in comparisons between the two methods (Aurora Prado, Steppe, Tavares, KedorHackmann and Santoro 2005, Carlucci, Anzini, Rovini, Cattaneo, Merlini and Tabucchi 2007, Clohs and McErlane 2003, Kocevar, Glavac, Injac and Kreft 2008, Kowalski and Plenis 2007, Muscarella, Iammarino, Centonze and Palermo 2005). Sometimes HPLC was not useful (Hilz, de Jong, Kabel, Schols and Voragen 2006), due to the physicochemical properties of the analyte resulting in poor resolution and reproducibility (Bexheti, Anderson, Hutt and Hanna-Brown 2006). 
Table 1 The within batch imprecision and inaccuracy of three quality controls together with LLOQ and ULOQ.

\begin{tabular}{llllll}
\hline Data & LLOQ & QC1 & QC2 & QC3 & ULOQ \\
\hline Nominal Conc $(\mu \mathrm{g} / \mathrm{ml})$ & 0.03 & 0.50 & 5.0 & 50.0 & 100.0 \\
\hline Mean & 0.03 & 0.51 & 4.86 & 47.19 & 95.97 \\
SD & 0.001 & 0.002 & 0.019 & 0.105 & 2.614 \\
CV\% & 4.02 & 0.41 & 0.40 & 0.22 & 2.73 \\
Inaccuracy \% & 3.40 & 2.65 & 2.83 & 5.63 & 4.25 \\
\hline
\end{tabular}

Table2 The between batch imprecision and inaccuracy of three quality controls together with LLOQ and ULOQ.

\begin{tabular}{llllll}
\hline Data & LLOQ & QC1 & QC2 & QC3 & ULOQ \\
\hline Nominal Conc $(\mu \mathrm{g} / \mathrm{ml})$ & 0.03 & 0.50 & 5.0 & 50.0 & 100.0 \\
\hline $\mathrm{n}=$ & 6 & 6 & 6 & 6 & 6 \\
\hline Mean & 0.03 & 0.51 & 5.03 & 48.46 & 92.29 \\
$\mathrm{SD}$ & 0.001 & 0.004 & 0.083 & 0.492 & 0.986 \\
$\mathrm{CV} \%$ & 3.66 & 0.85 & 1.65 & 1.02 & 1.07 \\
Inaccuracy \% & 4.38 & 1.61 & 1.41 & 3.46 & 7.71 \\
\hline
\end{tabular}


Table 3 Three freeze/ thaw cycles of the $100 \mu \mathrm{g} / \mathrm{mL}$ stock solutions.

\begin{tabular}{l|c}
\hline Data & Standard $100 \mu \mathrm{g} / \mathrm{ml}$ \\
\hline Time 0 & 100.78 \\
Freeze / thaw week 1 & 101.2 \\
Freeze / thaw week 2 & 99.79 \\
Freeze / thaw week 3 & 103.23 \\
CV\% & 1.42 \\
\hline
\end{tabular}

Table 4 The mean percentage error and the absolute mean percentage error between the HPLC and the CE measurements.

\begin{tabular}{l|cc}
\hline Data & MPE & AMPE \\
\hline Mean & 2.23 & 7.86 \\
SD & 8.08 & 4.63 \\
$\mathrm{n}$ & 102 & 102 \\
\hline
\end{tabular}


Figure 1 Chemical structures of tetracaine, procaine, and 4-butylaminobenzoic acid.<smiles>CCCCNc1ccc(C(=O)OCCN(C)C)cc1</smiles>

Tetracaine<smiles>CCN(CC)CCOC(=O)c1ccc(N)cc1</smiles>

Procaine<smiles>CCCCNc1ccc(C(=O)O)cc1</smiles>

4-butylaminobenzoic acid 
Figure2 A) Chromatograph obtained from extracted tape of time zero patient sample spiked with IS of $150 \mu \mathrm{g} / \mathrm{ml}$ procaine, displayed at $312 \mathrm{~nm}$.

B) Chromatograph of extracted tape of $10 \mu \mathrm{g} / \mathrm{ml}$ tetracaine spiked with IS of $150 \mu \mathrm{g} / \mathrm{ml}$ procaine, run under the optimum conditions and displayed at 312 $\mathrm{nm}$.

C) Chromatograph of extracted tape of 1 hour post-treatment spiked with IS of $150 \mu \mathrm{g} / \mathrm{ml}$ procaine, displayed at $312 \mathrm{~nm}$. 


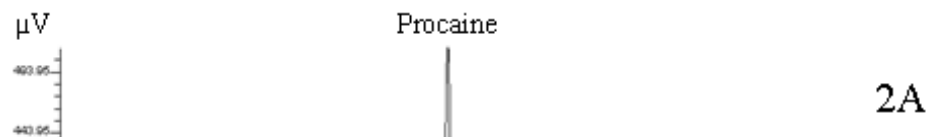

A
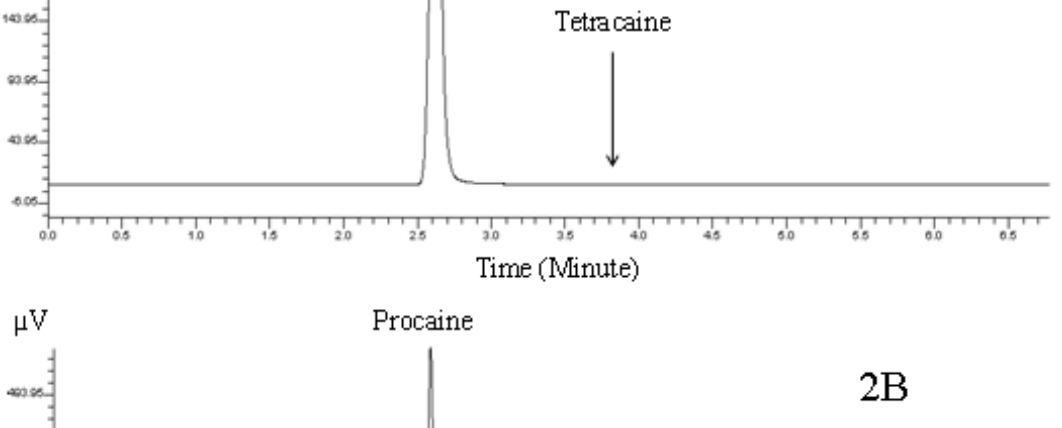

B

Tetracaine
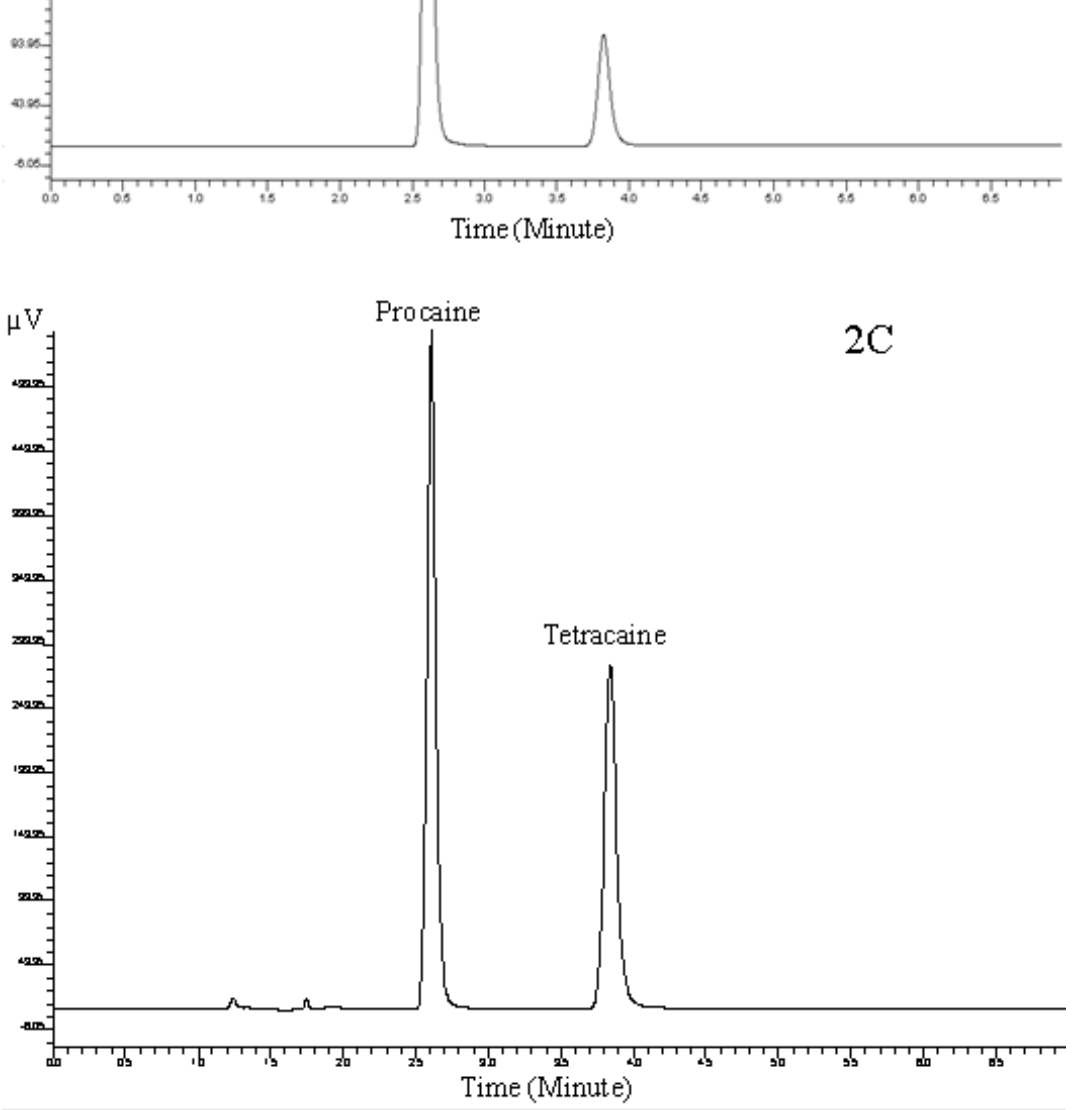
Figure 3 Typical calibration curve and linearity of tetracaine (HPLC).

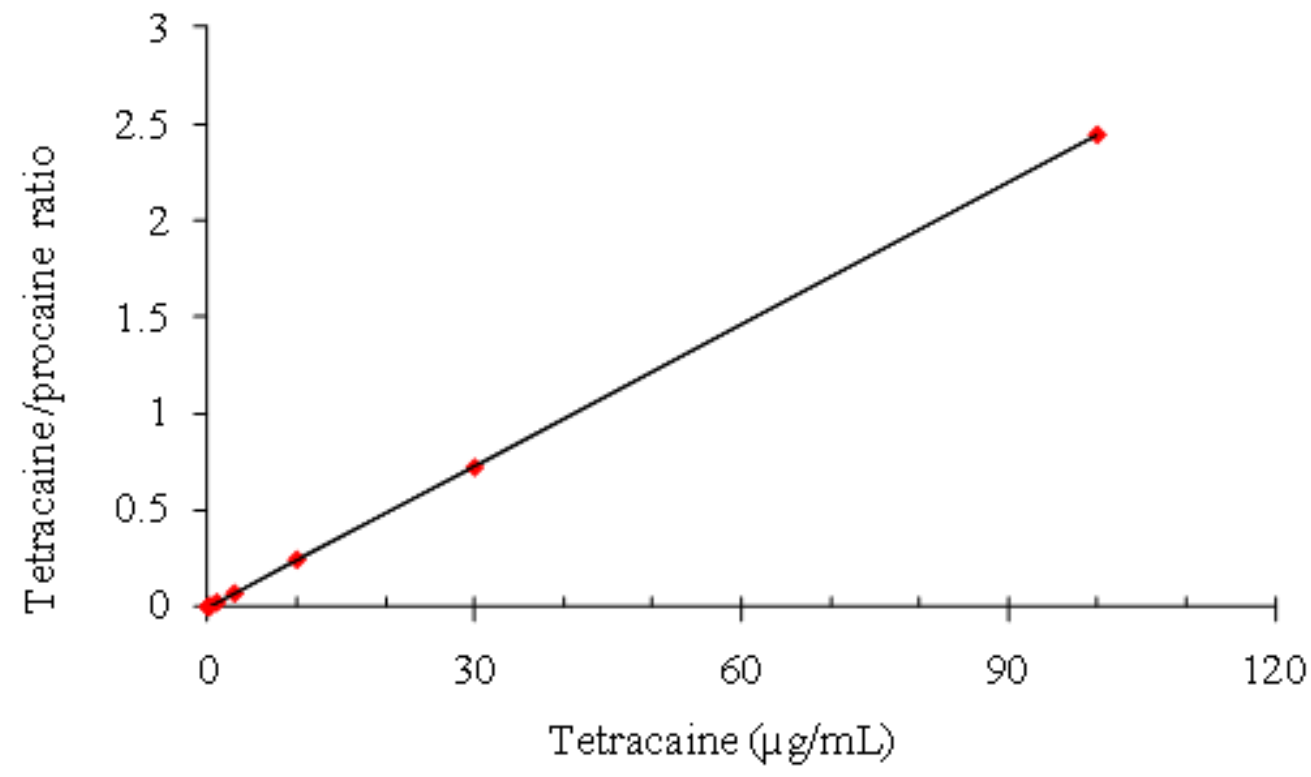


Figure 4 Bland-Altman analysis: correlation of HPLC and CE in the analysis of tetracaine tape stripping samples, $(\mathrm{SD}= \pm 8.018$, Mean $=2.231, \mathrm{n}=102)$.

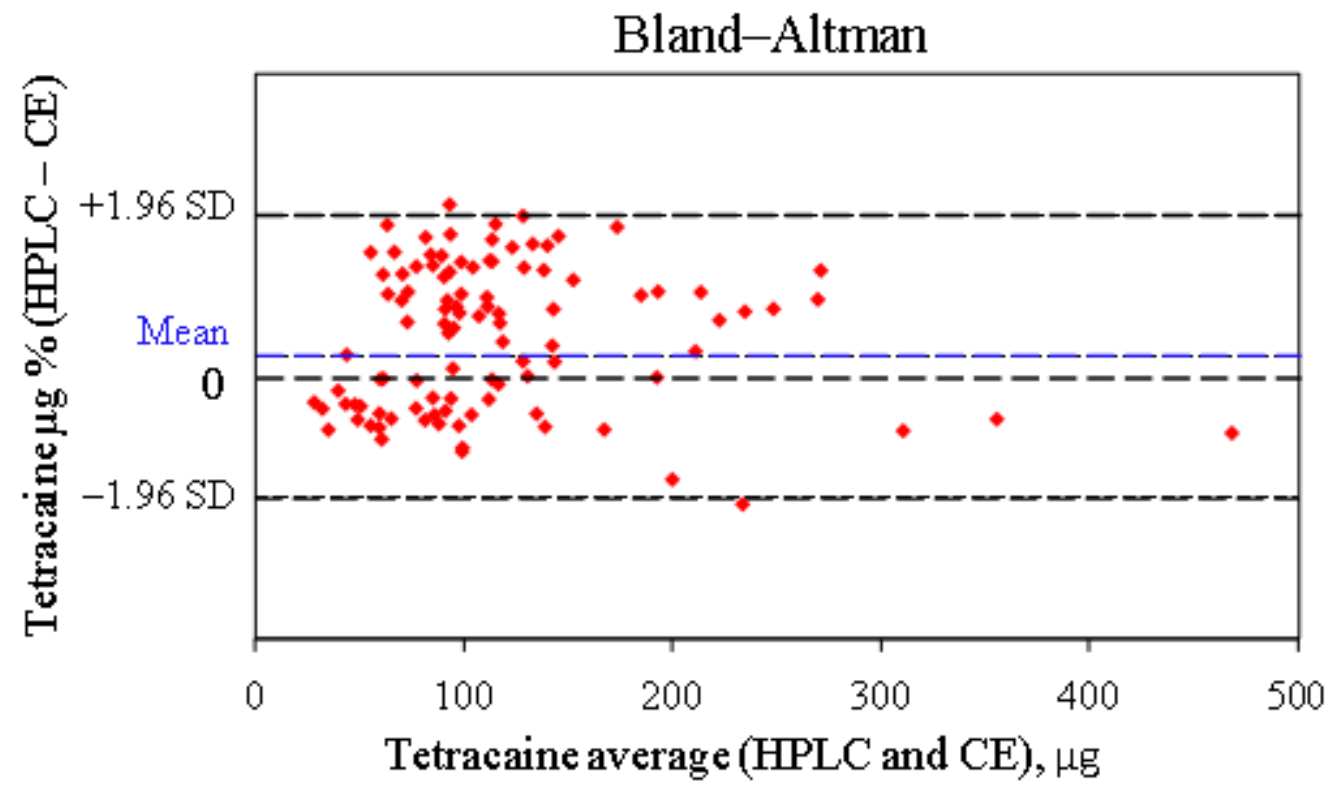




\section{References}

Al-Otaibi F, Tucker AT, Johnston A and Perrett D Rapid analysis of tetracaine for a tape stripping pharmacokinetic study using short-end capillary electrophoresis. Biomed Chromatogr 2009; 23 (5): 488-91. DOI: 10.1002/bmc.1142.

Aurora Prado MS, Steppe M, Tavares MF, Kedor-Hackmann ER and Santoro MI Comparison of capillary electrophoresis and reversed-phase liquid chromatography methodologies for determination of diazepam in pharmaceutical tablets. J Pharm Biomed Anal 2005; 37 (2): 273-9. DOI: 10.1016/j.jpba.2004.10.026.

Bareggi SR, Pirola R and De Benedittis G Skin and plasma levels of acetylsalicylic acid: a comparison between topical aspirin/diethyl ether mixture and oral aspirin in acute herpes zoster and postherpetic neuralgia. Eur J Clin Pharmacol 1998; 54 (3): 231-5.

Bexheti D, Anderson EI, Hutt AJ and Hanna-Brown M Evaluation of multidimensional capillary electrophoretic methodologies for determination of amino bisphosphonate pharmaceuticals. J Chromatogr A 2006; 1130 (1): 137-44. DOI:

10.1016/j.chroma.2006.06.110.

Bishai R, Taddio A, Bar-Oz B, Freedman MH and Koren G Relative efficacy of amethocaine gel and lidocaine-prilocaine cream for Port-a-Cath puncture in children. Pediatrics 1999; 104 (3): e31.

Boyd R and Jacobs M Towards evidence based emergency medicine: best BETs from the Manchester Royal Infirmary. EMLA or amethocaine (tetracaine) for topical analgesia in children. Emerg Med J 2001; 18 (3): 209-10.

Carlucci F, Anzini M, Rovini M, Cattaneo D, Merlini S and Tabucchi A Development of a CE method for the determination of mycophenolic acid in human plasma: a comparison with HPLC. Electrophoresis 2007; 28 (21): 3908-14. DOI: 10.1002/elps.200700190.

Clohs L and McErlane KM Comparison between capillary electrophoresis and highperformance liquid chromatography for the stereoselective analysis of carvedilol in serum. $\mathbf{J}$ Pharm Biomed Anal 2003; 31 (3): 407-12.

Drivas EI, Hajiioannou JK, Lachanas VA, Bizaki AJ, Kyrmizakis DE and Bizakis JG Cocaine versus tetracaine in septoplasty: a prospective, randomized, controlled trial. J Laryngol Otol 2007; 121 (2): 130-3. DOI: 10.1017/S0022215106002386.

FDA Guidance for industry bioanalytical method validation. 2001: 1 online resource (22 p.), availabe at http://www.fda.gov/downloads/Drugs/Guidances/ucm070107.pdf.

Hilz H, de Jong LE, Kabel MA, Schols HA and Voragen AG A comparison of liquid chromatography, capillary electrophoresis, and mass spectrometry methods to determine xyloglucan structures in black currants. J Chromatogr A 2006; 1133 (1-2): 275-86. DOI: 10.1016/j.chroma.2006.08.024.

ISO-5725 Accuracy (trueness and precision) of measurement methods and results. British Standards Institute 1994.

Kocevar N, Glavac I, Injac R and Kreft S Comparison of capillary electrophoresis and high performance liquid chromatography for determination of flavonoids in Achillea millefolium. J Pharm Biomed Anal 2008; 46 (3): 609-14. DOI: 10.1016/j.jpba.2007.11.016.

Kowalski P and Plenis A Comparison of HPLC and CE methods for the determination of cetirizine dihydrochloride in human plasma samples. Biomed Chromatogr 2007; 21 (9): $903-$ 11. DOI: $10.1002 / \mathrm{bmc} .818$.

Marin A and Barbas C Systematic comparison of different functionality columns for a classical pharmaceutical problem. J Pharm Biomed Anal 2006; 40 (2): 262-70. DOI: 10.1016/j.jpba.2005.07.028. 
Martindale W, Reynolds JEF and Parfitt K. The extra pharmacopoeia, Pharmaceutical Press: London, 1996.

Mazumdar B, Tomlinson AA and Faulder GC Preliminary study to assay plasma amethocaine concentrations after topical application of a new local anaesthetic cream containing amethocaine. Br J Anaesth 1991; 67 (4): 432-6.

McCafferty DF, Woolfson AD and Boston V In vivo assessment of percutaneous local anaesthetic preparations. Br J Anaesth 1989; 62 (1): 17-21.

Menon GN and Norris BJ Simultaneous determination of tetracaine and its degradation product, p-n-butylaminobenzoic acid, by high-performance liquid chromatography. J Pharm Sci 1981; 70 (5): 569-70.

Murtaza R, Jackman HL, Alexander B, Lleshi-Tali A, Winnie AP and Igic R Simultaneous determination of mepivacaine, tetracaine, and p-butylaminobenzoic acid by high-performance liquid chromatography. J Pharmacol Toxicol Methods 2001; 46 (3): 131-6.

Muscarella M, Iammarino M, Centonze D and Palermo C Measurement of histamine in seafood by HPLC, CE, and ELISA: comparison of three techniques. Vet Res Commun 2005; 29 Suppl 2: 343-6. DOI: 10.1007/s11259-005-0077-2.

Qin WW, Jiao Z, Zhong MK, Shi XJ, Zhang J, Li ZD and Cui XY Simultaneous determination of procaine, lidocaine, ropivacaine, tetracaine and bupivacaine in human plasma by high-performance liquid chromatography. J Chromatogr B Analyt Technol Biomed Life Sci 2010; 878 (15-16): 1185-9. DOI: 10.1016/j.jchromb.2010.03.003.

Woolfson AD, McCafferty DF and Boston V Clinical experiences with a novel percutaneous amethocaine preparation: prevention of pain due to venepuncture in children. Br J Clin Pharmacol 1990; 30 (2): 273-9. 